\title{
¿Son los Vinos de Pago la figura más eficiente entre las DOP españolas?
}

\author{
Fernando Vidal ${ }^{1}$, Jesús T. Pastor ${ }^{2}$, Juan Aparicio ${ }^{2}$, y Lidia Ortiz ${ }^{2}$ \\ ${ }^{1}$ Departamento de Economía Agroambiental, UMH. EPSO, Carretera de Beniel km 3,2, Orihuela 03312, España \\ ${ }^{2}$ Centro de Investigación Operativa, UMH, Edificio Torretamarit, Elche 03202, España
}

\begin{abstract}
Resumen. El Reglamento (CE) 1151/2012 del Parlamento Europeo y del Consejo, de 21 de noviembre de 2012, define, entre las figuras de protección para los productos agrícolas, las Denominaciones de Origen Protegidas (DOP). En España, los términos tradicionales utilizados para indicar que el producto se acoge a una DOP vitivinícola son: Denominación de Origen, Denominación de Origen Calificada, Vino de Calidad con indicación geográfica, Vino de Pago y Vino de Pago Calificado. Trabajos anteriores han analizado la eficiencia de las DOP, sin embargo, el caso de los Vinos de Pago, la figura más exclusiva y más reciente, no ha sido objeto de este tipo de análisis. El presente trabajo pretende ser una primera aproximación a la hora de analizar si esta figura presente comportamientos económicos diferenciados respecto al resto de figuras, y constatar si es la solución más eficiente en un mercado tan global y competitivo como el vinícola. Para ello se han tomado los últimos datos económico-productivos oficiales disponibles respecto a las DOP vitivinícolas españolas. Los resultados muestran que las diferentes figuras no presentan diferencias significativas excepto en el caso de las ventas por número de viticultores, donde los Vinos de Pago presentan valores superiores al resto de figuras.
\end{abstract}

\section{Introducción}

\subsection{Las figuras de protección}

Según el Reglamento (CE) 1151/2012 del Parlamento Europeo y del Consejo, de 21 de noviembre de 2012, sobre los regímenes de calidad de los productos agrícolas y alimenticios, una Denominación de Origen Protegida (DOP) vendría definida por: 1) Un nombre que identifica un producto, 2) Originario de un lugar determinado, una región o, excepcionalmente, un país, 3) Cuya calidad o características se deben fundamental o exclusivamente a un medio geográfico particular, con los factores naturales y humanos inherentes a él, y 4) Cuyas fases de producción tengan lugar en su totalidad en la zona geográfica definida [1]. La otra figura de protección que contempla el citado Reglamento es el de las Indicaciones Geográficas Protegidas (IGP). España cuenta en la actualidad con 90 DOP y 41 IGP.

En el caso de España, los términos tradicionales que se utilizan para indicar que el producto se acoge a una DOP vitivinícola son los siguientes: Denominación de Origen (DO), Denominación de Origen Calificada (DOCa), Vino de Calidad con indicación geográfica (VC), Vino de Pago (VP) y Vino de Pago Calificado (VPCa). Dichas figuras vienen definidas en la Ley 24/2003, de 10 de julio, de la Viña y del Vino (BOE 11-07-2003), y en particular para el caso de los VP, los define como los originarios de un pago "entendiendo por tal el paraje o sitio rural con características edáficas y de microclima propias que lo diferencian y distinguen de otros de su entorno, conocido con un nombre vinculado de forma tradicional y notoria al cultivo de los viñedos de los que se obtienen vinos con rasgos y cualidades singulares y cuya extensión máxima será limitada reglamentariamente por la Administración competente, de acuerdo con las características propias de cada Comunidad Autónoma, y no podrá ser igual ni superior a la de ninguno de los términos municipales en cuyo territorio o territorios, si fueren más de uno, se ubique". Se entiende que existe vinculación notoria con el cultivo de los viñedos, cuando el nombre del pago venga siendo utilizado de forma habitual en el mercado para identificar los vinos obtenidos en aquél durante un período mínimo de cinco años. En caso de que la totalidad del pago se encuentre incluida en el ámbito territorial de una denominación de origen calificada, podrá recibir el nombre de "pago calificado", y los vinos producidos en él se denominarán "de pago calificado", siempre que acredite que cumple los requisitos exigidos a los vinos de la DOCa y se encuentra inscrito en la misma [1].

\subsection{Los Vinos de Pago en España}

Como se ha comentado, la figura de los VP se reconoce a partir de la entrada en vigor de la Ley 24/2003. Desde entonces se han reconocido un total de 14 , lo que representa el 15,5\% del total de DOP. Señalar que la Comunidad Autónoma de Castilla - La Mancha concentra la mitad este tipo de vinos. En la Tabla 1 se muestra la superficie de los diferentes VP y el montante económico total comercializado por los mismos [1], señalando de éste el porcentaje comercializado en el mercado nacional.

El presente trabajo pretende ser una primera aproximación a la hora de analizar si esta figura presenta comportamientos económicos diferenciados respecto al resto de figuras, y así poder concluir si es la solución más eficiente en un mercado tan global y competitivo como el vinícola. 
Tabla 1. Los Vinos de Pago en España (2012/2013).

\begin{tabular}{|l|l|l|}
\hline & Ventas $(€)$ & Superficie (ha) \\
\hline Aylés & No disponible & 46 \\
\hline Calzadilla & $350.000(45,7 \%)$ & 13 \\
\hline Campo de la Guardia & $1.517 .024(72,3 \%)$ & 81 \\
\hline Casa del Blanco & $415.336(31,4 \%)$ & 92 \\
\hline Dehesa del Carrizal & $417.819(50,4 \%)$ & 26 \\
\hline Dominio de Valdepusa & $1.231 .280(44,0 \%)$ & 49 \\
\hline El Terrerazo & $132.816(71,5 \%)$ & 61 \\
\hline Finca Élez & $213.047(48,3 \%)$ & 39 \\
\hline Guijoso & $144.048(96,6 \%)$ & 59 \\
\hline Los Balagueses & $48.000(100,0 \%)$ & 18 \\
\hline Pago de Arínzano & $810.447(14,3 \%)$ & 128 \\
\hline Pago de Otazu & $84.400(38,7 \%)$ & 103 \\
\hline Pago Florentino & $531.859(50,5 \%)$ & 58 \\
\hline Prado de Irache & $37.533(91,3 \%)$ & 17 \\
\hline
\end{tabular}

( $)$ = Ventas en España /Total Ventas en porcentaje.

\section{Material y métodos}

La información relativa a los aspectos económicos y productivos de las DOP españolas se obtiene a partir de las últimas estadísticas publicadas por el Ministerio de Agricultura, Alimentación y Medio Ambiente y hacen referencia a la campaña 2012/2013 [1]. Para las diferentes figuras (DO, VC y VP) se han considerado los datos referidos a superficie (SUP, en hectáreas), número de viticultores (VIT), número de bodegas (BOD) y volumen comercializado (VENTAS, en euros).

Esta aproximación preliminar al análisis de la eficiencia de los VP españoles se abordará desde una doble perspectiva. En primer lugar se analizará desde un aspecto económico-productivo si determinados ratios presentan comportamientos diferenciados entre las tres figuras principales dentro de las DOP españolas. Para ello se realizará un análisis de la varianza para todas las DOP españolas para los siguientes ratios: Ventas/Superficie, Ventas/Número de Viticultores y Ventas/Número de Bodegas.

El segundo de estos análisis preliminares sería el cálculo de la eficiencia de las diferentes DOP mediante el empleo de métodos no paramétricos, en particular el empleo del Data Envelopment Analysis (DEA). Esta metodología ya ha sido empleada anteriormente en el análisis de las DO españolas [2,3], las IGP [4] o ambas conjuntamente [5]. Sin embargo, los VP no habían sido incluidos en estos análisis hasta la fecha.

En particular, se ha recurrido dentro del DEA al uso del modelo BCC con orientación input; modelo radial clásico (una explicación en mayor profundidad de la metodología DEA puede encontrarse en los trabajos antes mencionados [2-5]). Las variables empleadas como inputs han sido: VIT, SUP y VIT, mientas que se ha considerado en este caso un solo output: VENTAS.

\section{Resultados}

En el primero de los análisis se han considerado 77 DOP, descartando aquellas que no habían aportado los datos necesarios o estos eran incompletos. De ellas, 59 han sido DO, 13 han sido VP y 5 son VC.
Tabla 2. ANOVA variables económicas por DOP (2012/2013).

\begin{tabular}{|l|l|l|}
\hline Ventas $(€) /$ Superficie (ha) & Media & Desv. stand. \\
\hline DO & $6.916,56$ & $6.196,26$ \\
\hline VP & $9.433,96$ & $9.156,28$ \\
\hline VC & $5.300,92$ & $6.019,63$ \\
\hline Total $(\mathrm{F}=0,959)$ & $7.236,66$ & $6.752,56$ \\
\hline Ventas $(€) / \mathrm{N}^{\circ}$ de Viticultores* & Media & Desv. stand. \\
\hline DO & $21.847,15$ & $25.861,72$ \\
\hline VP & $409.074,54$ & $409.147,36$ \\
\hline VC & $65.812,30$ & $114.137,41$ \\
\hline Total $(\mathrm{F}=28,203)$ & $90.078,08$ & $220.650,95$ \\
\hline Ventas $(€) / \mathrm{N}^{\circ}$ de Bodegas & Media & Desv. stand. \\
\hline DO & $559.590,90$ & $707.953,72$ \\
\hline VP & $456.431,46$ & $466.638,38$ \\
\hline VC & $58.132,99$ & $28.761,23$ \\
\hline Total $(\mathrm{F}=1,405)$ & $509.612,17$ & $657.838,81$ \\
\hline
\end{tabular}

* Diferencias significativas al $99 \%$.

Tabla 3. Índices de eficiencia (IE) de las DOP (2012/2013).

\begin{tabular}{|l|}
\hline Grupo A. IE = 1 \\
\hline Calzadilla (VP), Campo de la Guardia (VP), Cangas (VC), \\
Cava (DO), Dominio de Valdepusa (VP), Rías Baixas \\
(DO), Ribeiro (DO), Rueda (DO) y Valdepeñas (DO) \\
\hline Grupo B. 1 $\geq$ IE $\leq 2$ \\
\hline Cariñena (DO), Jerez-Xérès-Sherry (DO), Dehesa del \\
Carrizal (VP), Pla i Llevant (DO), Somontano (DO), \\
Binissalem (DO), Campo de Borja (DO), Lebrija (VC), \\
Chacolí de Bizkaia (DO), Valdeorras (DO), Ribera del \\
Duero (DO), Yecla (DO), Chacolí de Álava (DO), Pago \\
de Arínzano (VP), Monterrei (DO), Priorat (DOCa) \\
\hline Grupo C. 2 $\geq$ IE $\leq 4$ \\
\hline Pago Florentino (VP), Málaga (DO), El Hierro (DO), \\
Condado de Huelva (DO), Cataluña (DO), Cigales (DO), \\
Bierzo (DO), Montilla Moriles (DO), Ycoden-Daute-Isora \\
(DO), Costers del Segre (DO), Toro (DO), Utiel-Requena \\
(DO), La Mancha (DO), Lanzarote (DO), Jumilla (DO), \\
Pla de Bages (DO), Casa del Blanco (VP), Ribeira Sacra \\
(DO) y Finca Élez (VP) \\
\hline Grupo D. IE $\geq 4$ \\
\hline Mondéjar (DO), Uclés (DO), Almansa (DO), Tierra de \\
León (DO), Valtiendas (VC), Sierras de Málaga (DO), La \\
Palma (DO), Tacoronte-Acentejo (DO), Manzanilla S.B. \\
(DO), Gran Canaria (DO), Arlanza (DO), Granada (VC), \\
Arribes (DO), Guijoso (VP), Bullas (DO), El Terrerazo \\
(VP), Abona (DO), Los Balagueses (VP), Valle de Güímar \\
(DO), Prado de Irache (VP), Ribera del Guadiana (DO), \\
Tarragona (DO), Pago de Otazu (VP), Tierra del Vino \\
de Zamora (DO), Valles de Benavente (VC), Manchuela \\
(DO), La Gomera (DO), Sierra de Salamanca (VC), Islas \\
Canarias (VC), Méntrida (DO) y Ribera del Júcar (DO) \\
\hline
\end{tabular}

Los ratios económicos nos muestran que no existen diferencias estadísticamente significativas entre los VP y el resto de figuras en el caso de las Ventas por Superficie o de Ventas por Número de Bodegas (Tabla 2). Sin embargo, sí que existen en el caso de las Ventas por Número de Viticultores, donde los valores medios para las VP son claramente superiores a los de las DO o los VC. Señalar que en el caso de los VP, tanto la variable VIT como BOD toma el valor uno. 
La aplicación del modelo BCC con orientación input a los tres inputs (SUP, VIT y BOD) y el output antes mencionado (VENTAS) nos permite clasificar las diferentes DOP en función de su eficiencia, de manera que un coeficiente 1 indicaría que esa DOP es eficiente, mientras que valores superiores a 1 reflejarían ciertas fuentes de ineficiencia. A efectos prácticos se han clasificado las diferentes DOP en 4 grupos de eficiencia (Tabla 3), desde las plenamente eficientes (Grupo A con un índice de eficiencia, IE, igual a 1) a las altamente ineficientes (Grupo D, IE $\geq 4$ ). El orden en el que aparecen listadas las DOP en los grupos B, C y D es de menor a mayor ineficiencia dentro de cada grupo.

\section{Conclusiones}

La proliferación en los últimos años de VP en España, figura que supone unas mayores exigencias productivas y de comercialización, podría ser una opción y una oportunidad en un mercado tan global y competitivo como lo es vitivinícola. Hemos tratado de comprobar de una manera previa y muy sencilla si a priori este tipo de DOP presenta un comportamiento económico diferenciado respecto al resto y si podríamos pensar también que muestran una mayor eficiencia. A este respecto señalar que los VP no presentan diferencias de comportamiento en cuanto a Ventas/SUP ni en Ventas/BOD. Si que existen claras diferencias en cuanto al nivel de VENTAS/VIT. En este tipo de figuras, donde únicamente existe registrado un solo viticultor (aunque también una sola bodega), sus ratios medios son muy superiores a las DO o los VC.
En cuanto al análisis de su eficiencia, el modelo empleado (BCC orientación input) no nos permite afirmar que los VP sean más eficientes que el resto de figuras, ya que pese a que tres de ellos aparecen dentro del grupo A, formado por las nueve DOP eficientes (Calzadilla, Campo de la Guardia y Domino de Valdepusa), también cabe señalar que cinco de ellas forman parte del grupo de DOP altamente ineficientes (Grupo D).

Este análisis previo deberá completarse en un futuro con una ampliación del horizonte temporal (número de campañas analizadas), tratando de incorporar nuevas variables económicas o productivas y empleando modelos más discriminantes, como por ejemplo los modelos acotados.

\section{Referencias}

[1] MAGRAMA, Disponible en: http://www . magrama. gob.es/es/alimentacion/temas/calidadagroalimentaria/calidad-diferenciada/dop/ (2015)

[2] J. Aparicio, F. Borrás, J.T. Pastor, F. Vidal, Eur J Oper Res. 231(2): 443-451 (2013)

[3] F. Vidal, J.T. Pastor, F. Borrás; D. Pastor, Span J Agric Res, 11(2): 294-304 (2013)

[4] F. Vidal, J.T. Pastor, J. Aparicio, D.B. López-Lluch. Actas de Horticultura 60: 34 (2012)

[5] J. Pastor, F. Vidal, F. Borrás, D. Pastor. The quality wine sector in Spain. An efficiency analysis. International Conference on Business and Information. Sapporo, Japan (2012) 